\title{
World Geographical Ontology Model
}

\author{
Enas M.F. El Houby \\ Systems \& Information Department, \\ Engineering Division, \\ National Research Centre, Dokki, \\ Giza, Egypt
}

\begin{abstract}
Recent days have witnessed an explosive growth in generating data in geographic domain which leads to increasing the applications of geographical information system (GIS). Due to this increasing of GIS, GIS faced the problem of effective management of the spatial information from different resources and in different forms. So, Geographical ontologies have been introduced to the geographical domain not only as a concept model which can represent objects on semantic and knowledge level, but also to integrate geographical data from different sources and in different forms for reasoning and to facilitate knowledge sharing, in addition to assist in recognizing spatial terms.

In this research, the World geographical ontology model has been developed to meet the needs of the recognition of the terminologies and the semantic relationships between geographical terms related to any location in the world. It will enable search engine to perform a spatially aware search. The design of World ontology model has been developed based on the analysis and finding relations between different parts of World locations to adopt ontology concepts. Logical formulation has been used to describe the ontology to be able to infer more intelligent relations. Then the model has been implemented using protégé tool, a sample of related data has been collected and finally the system has been tested. Query answers integrate data from different sources.
\end{abstract}

\section{Keywords}

GIS, Geo-Ontology, OWL, Semantic Web

\section{INTRODUCTION}

A large portion of web resources may be regarded as geographically located. For example, many Web documents report on activities that take place in some locations on the Earth's surface. However, existing web facilities are poorly adapted to find information that is related to a particular location. When supplied with a spatial query, a typical search engine only returns web pages that include that place name involved. Other web documents that are associated to that place, e.g. that refer to places in the proximity of the subject place or are contained within it, may also be of interest but usually not returned [1]. That results in increasing interest in the geographic information system.

In geographic information system (GIS), great varieties of information from different domains are involved in order to solve actual problems. Usually spatial information is stored in diverse spatial databases, manipulated by different GIS platforms. Besides, different experts from various domains have different emphases and understanding even on the same GIS data set, i.e. semantic heterogeneity [2]. All these lead to different GIS cannot share and manipulate data effectively. Therefore, it is critical to deal with the problem of spatial information interoperability on semantic level. Ontology can describe the concept model of objects on semantic and knowledge level. When formalized, ontology can be interpreted by computer easily, and the information contained in the ontology can be processed on semantic level effectively. According to these reasons, ontology is introduced into GIS as a concept model which represents objects on semantic and knowledge level to solve these problems [3].

Geographical Ontology (Geo-ontology) is defined as the formalization of concepts shared among the GIS domain. It provides a controlled vocabulary or conceptualization for geographical concepts and supplies a shared understanding of knowledge among geographical communities. It results from analysis and modeling of ontology in geo-spatial application. It can be represented as concepts and relationships between the concepts which are abstracted from real geographic space. Geoontology does not only describe spatial data using the semantic encoding method to be more easily understood by computers, but also integrates geographical data coming from different sources and in different forms for reasoning. After formalization, geo-ontology is readable to both humans and computers $[3,4]$

In this research a world geographical ontology has been designed and constructed to assist in finding any geographical information related to a particular location. Logical formulation has been used to describe the ontology to be able to infer more intelligent relations.

The remainder of this paper is organized as follows. In section 2 , an overview for the previous related works is presented. In section 3, the world Geographical Ontology Model is described. In section 4, experimental results and queries application of world geographical ontology is briefly presented, before drawing conclusions and future work in section 5 .

\section{RELATED WORK}

A lot of previous works and issues related to semantic web, geo-ontology and geographical information systems which assist spatial search have been recognized. Krzysztof Janowicz [5] proposed an observation-driven ontology engineering framework, which shows how its layers can be realized using specific methodologies, and relates the framework to existing work on geo-ontologies. Jinghai Xu,et al. [6] proposed a conceptual model of knowledge for earthquake disaster emergency response (EDER). Geo-ontology is used to build basic modeling primitives and represent geospatial characteristics of the EDER knowledge. It provides a foundation for intelligent emergency response that helps to solve knowledge problems that improve earthquake disaster response. Martínez et al. [7] proposed geo-ontology design patterns which focused on content patterns, i.e., design solutions for domain classes and relationships. They proposed a logical pattern that addresses a frequent modeling problem that has hampered the development of sophisticated geo-ontologies 
in the past, namely how to model the quantification over types. They argued for the need for such a pattern, explained why it is difficult to model, demonstrated how to implement it using the Web Ontology Language OWL, and finally show how it can be applied to modeling concepts such as biodiversity. Sun, et al., [8] summarized the concept and connotation of Geo-ontology by analyzing the related international up-to-date documents and reports. The necessity of this research, especially probe into the Geo-ontology central problems and its construction methods, so as to provide some reference for further researches on Geoontology. Bharambe, et al. [9] focused on analogy reasoning using geo-ontology matching. A framework is proposed for analogy reasoning. Using an example of road networks, it is explained how analogy can be formulated, and then transferable and learnable patterns are generated.

Sinha, G., et al. [10] focused on the vision of Linked Topographic Data (LTD) which is critical for the semantic web. LTD involves a synergy between a wide variety of topographic information and services, but at its core is the theme of terrain, i.e., the shape of the earth's surface. Li, Wenwen, et al. [11] developed a semantic search tool that supports the intelligent discovery of polar datasets. This tool is built on latent semantic analysis technique, which improves search performance by identifying hidden semantic associations between terminologies used in the various datasets' metadata. Kalbasi, Reza, et al. [12] presented a two-step methodology and implementation to foster collaborative ontology engineering in the geo-sciences. The approach consists of the development of a minimalistic core ontology acting as a catalyst and the creation of a virtual collaborative development cycle. Both methodology and prototypical implementation have been tested which addresses environmental protection with respect to forests and climate change. Campelo el al., [13] presented a knowledge representation approach to model and manipulate spatiotemporal data. The geographic features which can be changed over time had been represented, since an appropriate modeling of these dynamic features can provide a natural way of defining other dynamic entities of geographic space, such as events and processes. Zhu, X. et al., [14] proposed a novel conceptual framework of direction relations in order to formalize the semantics and implicit information of direction relations, and present an extraction algorithm of implicit information, which produce a complete query instance of direction relations. Gimenez, Paulo JA et al. [15] presented an approach to the creation of a geo-ontology information model for representing geographic objects that represents the involved concepts, their characteristics and their relationships within the context of the Brazilian National Spatial Data Infrastructure (INDE) using the existing geographical names, in order to consolidate aspects of spatial metadata, spatial features and geographic names, as well as enabling the discovery and integration of geo-information. Ying Xia, et al. [16] developed, a novel retrieval method for Multimodal Point of Interest (MPOI) which is the core content of mobile geographic information service, Geo-ontology model were adopted to preprocess the MPOI data.

Sinha, Gaurav, et al. [17] developed the Surface Water ontology design pattern both for domain knowledge distillation and to serve as a conceptual building-block for more complex or specialized surface water ontologies. A distinction is made in this ontology between landscape features that act as containers (e.g., stream channels, basins) and the bodies of water (e.g., rivers, lakes) that occupy those containers. The pattern is implemented in OWL, and Description Logic axioms and a detailed explanation is provided. The practical value of the pattern in semantic querying of surface water datasets is illustrated through an annotated geospatial dataset and sample queries using the classes of the Surface Water pattern.

\section{THE WORLD GEOGRAPHICAL ONTOLOGY MODEL}

In this research, world geographical ontology model has been developed to answer queries about geographical information related to any particular location. First, the problem has been analyzed and the design of ontology model has been developed according to the analysis. Then the logic form has been used to formulate the model in a form suitable to infer more relations.

\subsection{Analysis and design of the World Geographical Ontology Model}

By analyzing the world geography, different entities and attributes that can be used to describe any world's geographical location have been collected. And by analyzing the meaning of each entity and its relation with the other entities, the set of concepts which can be used to represent the world geographical ontology and the relations with the other concepts are defined. The analysis identifies conceptual structure among these concepts which leads to an ontology that has these concepts described in a way suitable for knowledge exchange. So, the concept should be described by its position in the taxonomical hierarchy, concepts' attributes and also by relations that can be applied to the concepts. The relation can also be described by concepts that can be related together by this relation.

It was found that, any location in the world can be described by its climatic region, geographical region, and terrains which exist in the specified location. Resources and products which are found in the location represent an asset for it. Also human strains and activity of the people who live in it differ according to the specified location. So each of these 7 entities are represented as a class in world geographical ontology design as shown in Figure1, and they are used to describe any specified location in the world. The root of the hierarchy is defined as "Location" class to represent any searched location in the world. Each of these 7 classes can be divided into sub-classes. For example the "Geographical region" class can be divided into water body such as sea, ocean, stream and so on, and land body such as Continent, country, city and so on as shown in Figure2. The same for different classes can be divided to their sub-classes and each of sub-classes can further be classified but for simplicity and readability of the ontology the degree of granularity is limited to four levels, also not all sub-classes for specific class are represented (i.e. branching is limited). As depicted in Figure3, these classes are arranged in a hierarchy or taxonomy with the top class is "Location".

The various relations which can be investigated to relate different classes of the world geographical ontology (object properties) are defined as shown in table 1. As an example "Distrbuted_In" describes the resources which are distributed in the specified geographical region; it is identified for each instance of the class "Resources", the possible values that are suitable for the resources to be distributed in is any "GeographicalRegion". The inverse of "Distrbuted_In" is "Contain" which describes the geographical region that contains any specified resources; it is identified for each instance of the class "GeographicalRegion", the possible values that are suitable for the geographical region to contain is any "Resources".

The set of attributes for the ontology which are used to describe classes is shown in table 2. As an example "Synonyms" attribute describes the different names of any geographical location related items, such as different places' names; if 
variant names refer to the same place. Its possible values are any string value.

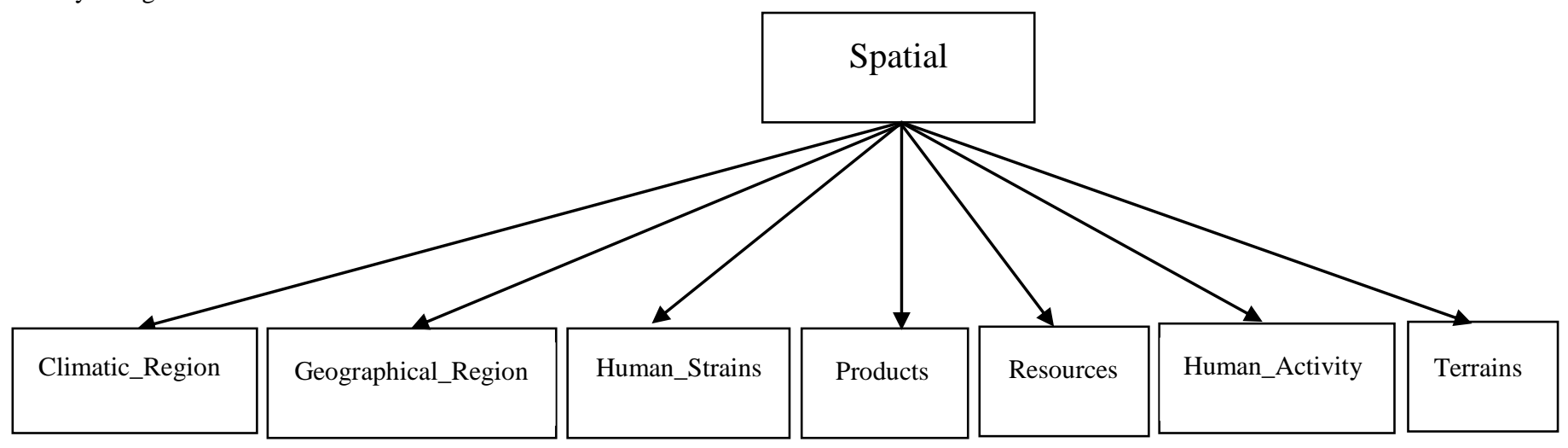

Figure 1: The main classes of World Geographical ontology

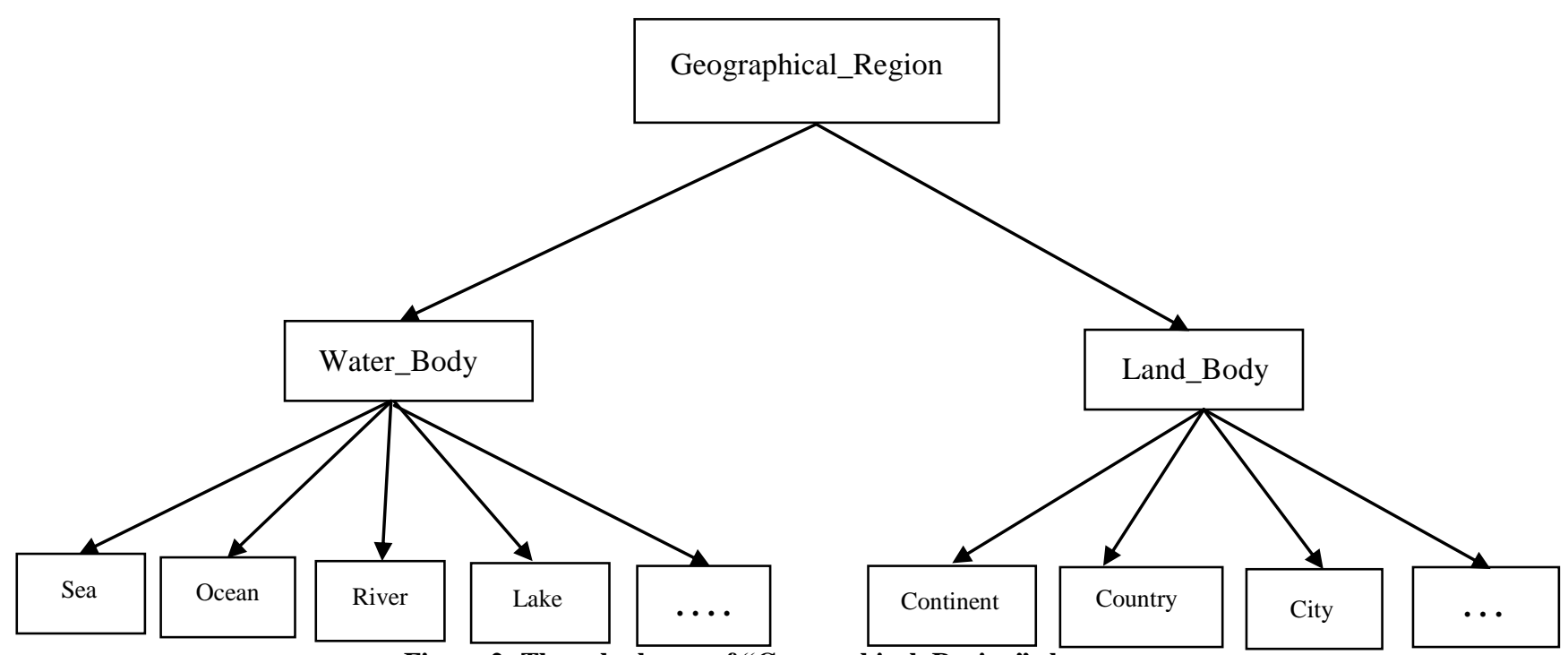

Figure 2: The sub-classes of "Geographical_Region" class 


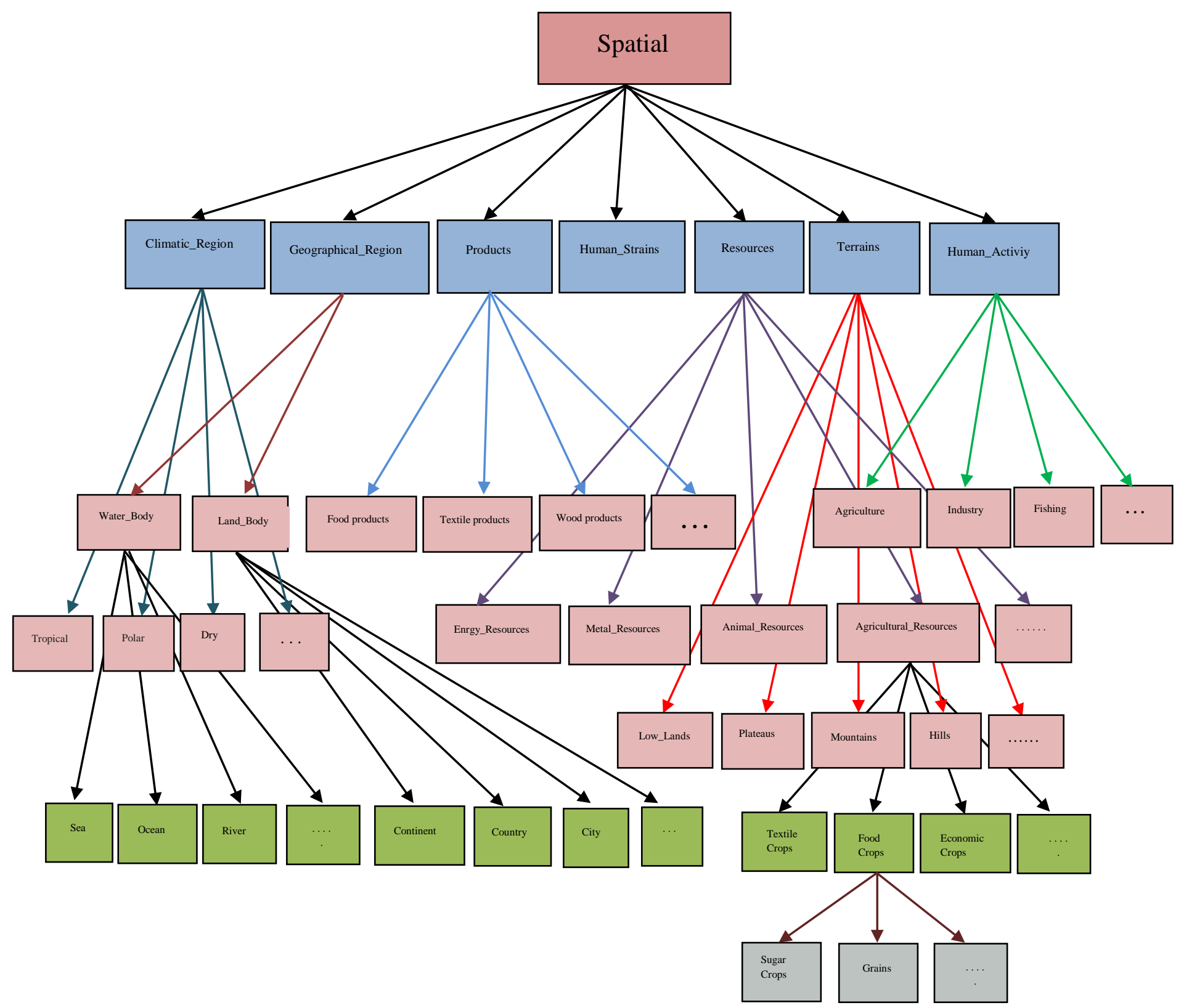

Figure 3: the class hierarchy of the World Geographical Ontology

Table 1: the set of object properties for World Geographical Ontology

\begin{tabular}{|l|l|l|l|}
\hline Object Properties & Domain Range & Range Domain & Inverse \\
\hline Distrbuted_In & Resources & GeographicalRegions & Contain \\
\hline Include & Continent & Country, City & Inside \\
\hline Comprise & Country & City & Is_part_of \\
\hline DependOn & HumanActivity & Resources, GeographicalRegions & affect \\
\hline Produce & Resources & Products & Produced_By \\
\hline Live_In & Human_Strains & Geographical_Region & Habited_By \\
\hline Involve & GeographicalRegions & Terrain & Lie_in \\
\hline Specified_By & GeographicalRegions & Climatic_Region & Specify \\
\hline East_Border & GeographicalRegions & GeographicalRegions & Wes_Border \\
\hline North_Border & GeographicalRegions & GeographicalRegions & South_Border \\
\hline NorthEast_Border & GeographicalRegions & GeographicalRegions & SouthWest_Border \\
\hline NorthWest_Border & GeographicalRegions & GeographicalRegions & SouthEast_Border \\
\hline
\end{tabular}


Table 2: The set of attributes for World Geographical Ontology

\begin{tabular}{|l|l|l|l|}
\hline Attribute & Domain & Range & Allowed values \\
\hline Synonyms & Location & String & \\
\hline Latitude_North & GeographicalRegions & String & \\
\hline Latitude_South & GeographicalRegions & String & \\
\hline Longitude_East & GeographicalRegions & String & \\
\hline Longitude_West & GeographicalRegions & String & \\
\hline Area & GeographicalRegions & String & \\
\hline Quantity & Products & String & \\
\hline Height & Terrain__Strains & String & \\
\hline Hair_color & Human_String & Fair, Dark \\
\hline Complexion & Human_Strains & String & Black, White \\
\hline Characteristics & Human_Strains & String & \\
\hline
\end{tabular}

\subsection{Representation of the World Geographical Ontology Model Using Logical Form}

An effective reasoning mechanism based on ontology can help to discover more exact and more valuable knowledge. The formulation of the ontology in logic form enables to infer different relations and constraints and perform more deep queries. So the World geographical ontology will be formulated in logic form to extract and infer more intelligent knowledge from the ontology. The World ontology will be defined in a logical form depending on Stumme et al. [18]. However, the definitions are freely modified to serve the aims of this research.

Stumme et al. [18] had used logical form for describing ontologies. Based on Stumme et al. the ontology $\mathrm{O}$ is a structure which can be described as follows:

$\mathrm{O}:=\{\mathrm{C},<\mathrm{c}, \mathrm{R}, \sigma \mathrm{r},<\mathrm{R}, \mathrm{A}, \sigma \mathrm{a}, \tau\}$

Where $\mathrm{C}, \mathrm{R}, \mathrm{A}$ and $\tau$ are sets whose elements are class identifiers, relation identifiers, attribute identifiers and data types, respectively. <c is called class hierarchy or taxonomy, and is used with the elements of $\mathrm{C}$ to describe which class is the super-class and /or subclass of the other classes. A function or is called relation signature, and it is used to describe the elements of R. A partial order $<R$ is called relation hierarchy, and is used to describe the dependence of relations of $\mathrm{R}$ on each other. A function $\sigma \mathrm{a}$ is called attribute signature, and is used to describe the elements of A. $\tau$ is a set of data types such as strings, integers, etc.

If $\mathrm{C} 1<\mathrm{c} C 2$, for $\mathrm{C} 1, \mathrm{C} 2 \in \mathrm{C}$, then $\mathrm{C} 1$ is a sub-class of $\mathrm{C} 2$, and $\mathrm{C} 2$ is a super-class of $\mathrm{C} 1$.

If $\mathrm{C} 1<\mathrm{c} \mathrm{C} 2$ and there is no $\mathrm{C} 3 \epsilon \mathrm{C}$ with $\mathrm{C} 1<\mathrm{c} \mathrm{C} 3<\mathrm{c} C 2$, then $\mathrm{C} 1$ is a direct sub-class of $\mathrm{C} 2$, and $\mathrm{C} 2$ is a direct super-class of $\mathrm{C} 1$ which can be denoted as $\mathrm{C} 1<\mathrm{D} C 2$.

If $\mathrm{C} 1<\mathrm{c} C 2$ and there is $\mathrm{C} 3 \in \mathrm{C}$ with $\mathrm{C} 1<\mathrm{c} C 3<\mathrm{c} C 2$, then $\mathrm{C} 1$ is an indirect sub-class of $\mathrm{C} 2$, and $\mathrm{C} 2$ is an indirect super-class of $\mathrm{C} 1$ which can be denoted as $\mathrm{C} 1<\mathrm{I} \mathrm{C} 2$.

\subsubsection{Logical Description of the Classes of World Geographical Ontology}

The World ontology is a structure which includes the following set $\mathrm{C}$ of classes:

C := \{Location, Climatic_Region, Tropical, Polar, Dry, Moderate, Continental, Geographical_Region, Land_Body, Continent, Country, City, Water_Body, Lake, Ocean, River,
Sea, Stream, Products, Food_products, Textile products, Wood products, Human_Strains, Resources, Fish_Resource, Enrgy_Resources, Metal_Resources, Animal_Resources, Agricultural_Resources, Textile Crops, Economic Crops, Food Crops, Sugar Crops, Grains, Terrains, Low_Lands, Plateaus, Mountains, Hills, Human_Activity, Agriculture, Industry, Fishing\}.

As depicted in Figure3, these classes are arranged in a hierarchy or taxonomy with the root is the "Location" class.

The sets of direct sub-class $<\mathrm{D}$ can be described logically as follows:

\{Climatic_Region, Geographical_Region, Products, Human_Strains, Resources, Terrains, Human_Activity $\}<D$ Location

\{Tropical, Polar, Dry, Moderate, Continental\} <D Climatic_Region

$\{$ Land_Body, Water_Body $\}<\mathrm{D}$ Geographical_Region

$\{$ Continent, Country, City\} <D Land_Body

$\{$ Lake, Ocean, River, Sea, Stream $\}<D$ Water_Body

\{Food_products, Textile products, Wood products $\}<D$ Products

\{Enrgy_Resources, Metal_Resources, Animal_Resources, Agricultural_Resources, Fish_Resource $\}<$ D Resources

\{Textile Crops, Economic Crops, Food Crops $\}$ D Agricultural_Resources

\{Sugar Crops, Grains $\}$ D Food Crops

$\{$ Low_Lands, Plateaus, Mountains, Hills $\}<D$ Terrains

\{Agriculture, Industry, Fishing\} <D Human_Activity

Where the direct sub-class

\{Continent, Country, City\} <D Land_Body

Means that the three classes \{Continent, Country, City\} are direct sub-classes of the class Land_Body. In other words, the Land_Body class is a direct super-class of the three classes \{Continent, Country, City\}.

The set of indirect sub-class in the ontology are:

\{Continent, Country, City, Lake, Ocean, River, Sea, Stream $<$ I Geographical_Region

$\{$ Sugar Crops, Grains $\}<$ I Agricultural_Resources 


\section{$\{$ Textile Crops, Economic Crops, Food Crops $\}<$ I Resources}

$\{$ Tropical, Polar, Dry, Moderate, Continental, Land_Body, Continent, Country,City, Water_Body, Lake, Ocean, River, Sea, Stream, Food products, Textile products, Wood products, Fish_Resource,Enrgy_Resources,Metal_Resources,Animal_Res ources,Agricultural_Resources,Textile_Crops,Economic_Crops , Food_Crops, Sugar_Crops, Grains, Low_Lands, Plateaus, Mountains, Hills, Agriculture, Industry, Fishing $\}<$ I Location

Where the indirect relation

\section{\{Sugar Crops, Grains $\}<$ I Agricultural_Resources}

Mean that the two classes \{Sugar Crops, Grains\} are indirect sub-classes of the class Agricultural_Resources. In other words, the Agricultural_Resources class is an indirect super-class of the two classes \{Sugar Crops, Grains .

Accordingly, the set of class hierarchy $<\mathrm{c}$ which include direct $<\mathrm{D}$ and indirect $<\mathrm{I}$ sub-class is defined as:

$<\mathrm{c}:=<\mathrm{D} \mathrm{U}<\mathrm{I} \quad$ Where $\mathrm{U}$ means union.

Each of the following sets of classes is disjoint:

Set $1:=$ (Land_Body, Water_Body)

Set $2:=($ Lake, Ocean, River, Sea, Stream)

Set3:= (Fish_Resource, Enrgy_Resources, Metal_Resources, Animal_Resources, Agricultural_Resources)

Set $4:=$ (Textile Crops, Food Crops)

Set5 $:=$ (Sugar Crops, Grains)

Set6 $:=$ (Food_products, Textile products, Wood products)

Set $7:=($ Low_Lands, Plateaus, Mountains, Hills)

Disjoint is denoted by “ $\neg$ ”, accordingly the last of the above disjoint sets must fulfill the following:

$\forall \mathrm{x}\{$ Low_Lands(x) ᄀ (Plateaus (x), Mountains (x), $\operatorname{Hills}(\mathrm{x}))\}$

and $\forall$ x \{ Plateaus (x) ᄀ (Low_Lands(x), Mountains (x), Hills(x))\}

and $\forall \mathrm{x}\{$ Mountains (x) ᄀ (Low_Lands(x), Plateaus (x), Hills(x))\}

and $\forall$ x \{ Hills $(\mathrm{x}) \neg$ (Low_Lands(x), Plateaus (x), Mountains $(\mathrm{x}))\}$

where the disjoint relation:

$\forall$ x \{ Low_Lands(x) ᄀ (Plateaus (x), Mountains (x), Hills(x)) $\}$ means that every element $\mathrm{x}$ which belongs to the class Low_Lands can't belong to the classes Plateaus or Mountains or Hills and so on for the other disjoint sets.

\subsubsection{Logical Description of the Attribute and Object Properties of World Ontology}

The set of relations between classes (object properties) in the ontology is defined as follow:

$\mathrm{R}:=\{$ Distrbuted_In, Contain, Include, Inside, Comprise, Is_part_of, DependOn, affect, Produce, Produced_By, Live_In, Habited_By, involve, Lie_in, Specified_By, Specify, East_Border, Wes_Border, North_Border, South_Border, NorthEast_Border, SouthWest_Border, NorthWest_Border, SouthEast_Border\}

The signature or of object properties is defined as follow: or $($ Comprise $)=($ Country, City $) \Leftrightarrow \sigma r($ Is_part_of $)=($ City, Country)

The object property "Comprise" has inverse property in the model named "Is part of".
Where

$\sigma r($ Comprise $)=($ Country, City $)$ means that Country "Comprise" City. The property "Comprise" has domain Country and range City. The inverse property of "Comprise" is "Is_part_of". The Domain of the property "Is_part_of" is City and the range is Country. The property and its inverse is written as follows:

or $\quad($ Comprise $)=($ Country, City $) \Leftrightarrow \sigma r($ Is_part_of $)=$ (City, Country)

Where the bidirectional arrow " $\Leftrightarrow$ " means 'inverse of'. The domain of property "Comprise" is the range of property "Is_part_of" and vice versa. The full set of object properties is shown in table1.

The set of attributes are:

A := \{Latitude_North (), Latitude_South (), Longitude_East, Longitude_West, Synonyms, Area, Quantity, height, Hair_color, Complexion, Characteristics

The following attributes have attribute signatures $\sigma a:$ $\sigma \mathrm{a}($ Characteristics $)=($ Human_Strains, string $)$ $\sigma a($ Synonyms $)=$ (Location,, string $)$

The "Synonyms" attribute describes the different names for the same individual for various classes; it can take any string value. The full set of attributes is shown in table2.

\section{EXPERIMENTAL RESULTS}

Different languages and tools have been used for representing and modeling ontologies. One of these tools is a visual ontology modeling software namely Protégé 3.4.6 developed by Stanford university [19] which is built on the top of OWL language. Protégé 3.4.6 has been used to build the World geo-ontology according to the proposed design. Different classes and properties have been created. Both data type properties (attributes) which describe classes and object properties which describe the relations between classes are created. Different conditions and disjoints classes have been specified. According to Protégé Tool, the root class of the ontology which is called "Location" is mapped to the root class 'Thing'. A snapshot of World Ontology developed using the Protégé software is shown in Figure 4.

A sample of OWL for Egypt description as an example of individual location which is represented in the ontology is shown in Figure 5. Egypt is defined as an instance under the class 'Country' which includes a 'City' instance called Cairo. Also a data type properties "Latitude_North" and "Latitude South" show that it lies between $31 . \overline{5} \mathrm{~N}$ and $22 \mathrm{~N}$ inside Africa continent as defined by object property "Inside". The object properties "East Border" and "South Border" show that red sea is the east border of Egypt and Sudan is the south border of Egypt.

The Query tool provided by Protege_3.4.6 is used to run the queries. Figure 6 shows a snapshot of the query $(\mathrm{Q})$ tool on Protégé. The following are samples of the queries which can be applied using World Geo-ontology.

Q1: Include (Continent, Canada) := $\{$ North_America $\}$

Where Include is the object property which specify the relation between continent and country and is used to find continent which include the searched country. In the above query "Canada" is the country under investigation, Continent is the domain where the query searches, and \{North_America\} is the result of the query. 
Q2: Inside (Country, North_America) $:=\{$ United_States, Canada, Mexico

Where in this case, Inside is the object property which is used to find country which inside the searched Continent. "North_America" is the continent under investigation, Country is the domain where the query searches, and the list of countries in the R.H.S \{United_States, Canada, Mexico\} is the result of the query. Q2 is the inverse of Q1.

Q3: Distrbuted_In (Sugar_Crops, Egypt) $:=$ \{Sugar_Cane, Beet\}
Q4: Comprise (Country, Aswan) := $\{$ Egypt $\}$

Q5:South_Border(Geographical_Region,Tunisia):= $\{$ Mediterrane_sea $\}$

Q6: Longitude_East (Country, $37 \mathrm{E}):=\{$ Egypt $\}$

Q7: Longitude_West (Country, 25 E):= $\{$ Egypt $\}$

Q8: Involve (Country, Himalayan):= $\{$ India $\}$

Q9: Height (Mountains, $8848 \mathrm{~m}):=\{$ Himalayan $\}$

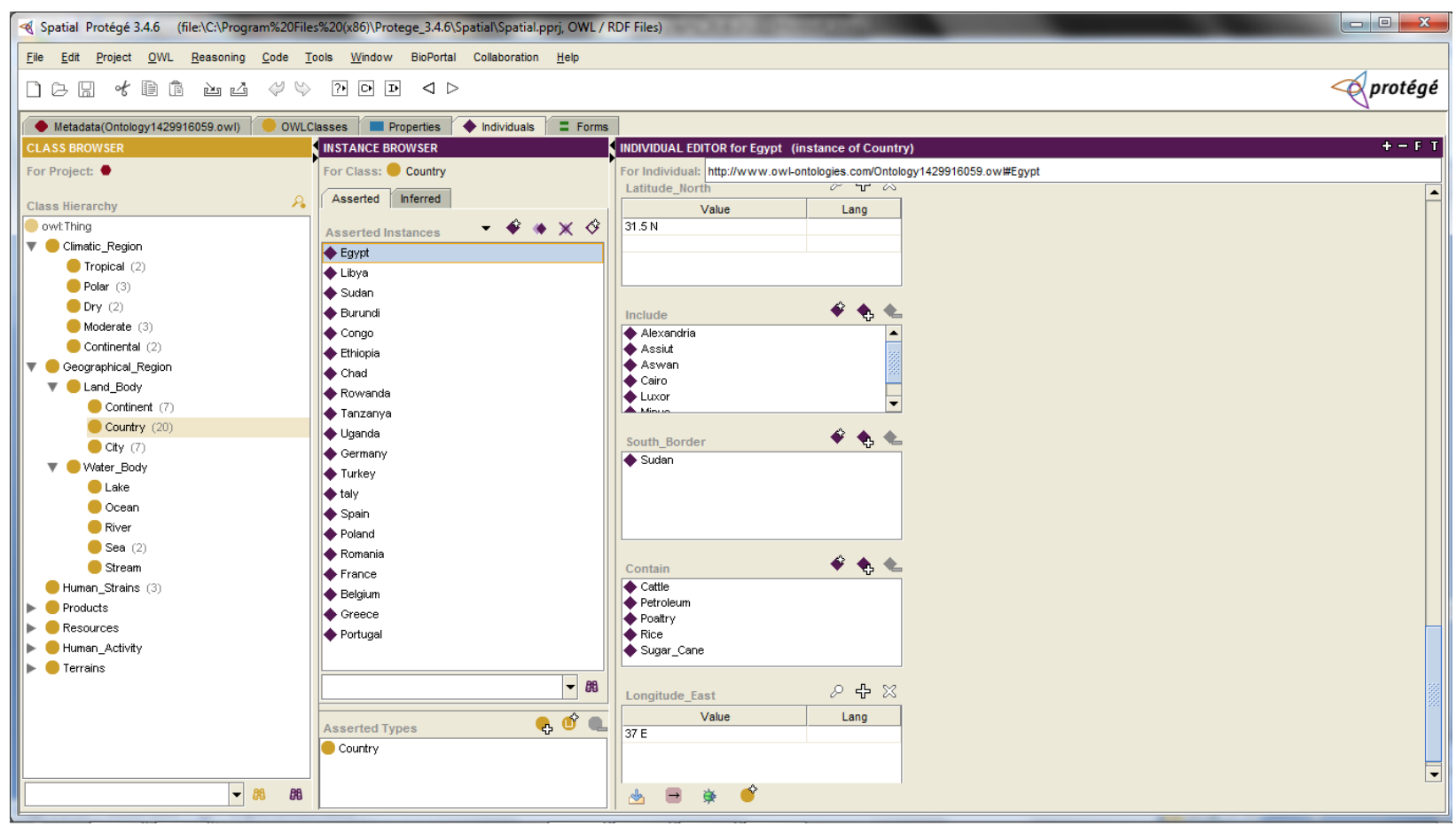

Figure 4: A snapshot of the Protégé window for the World Geographical ontology

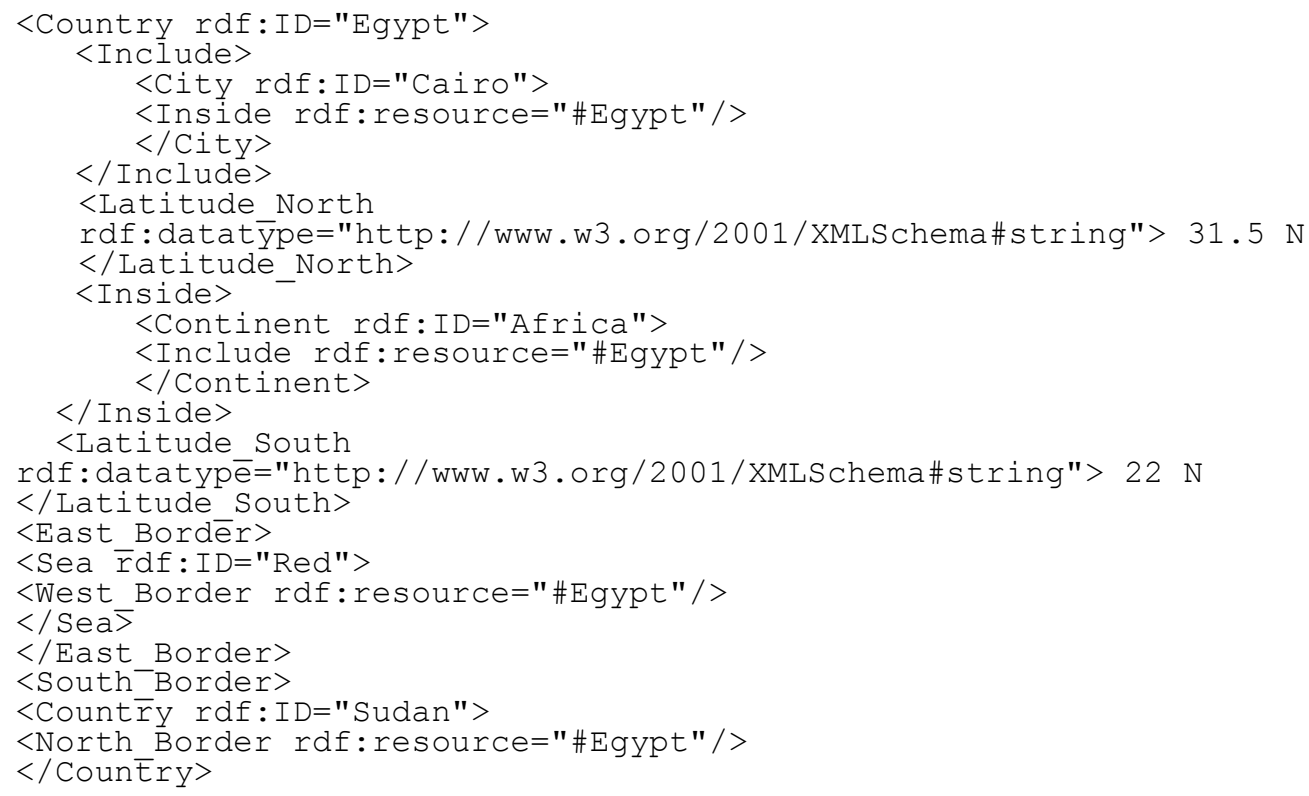




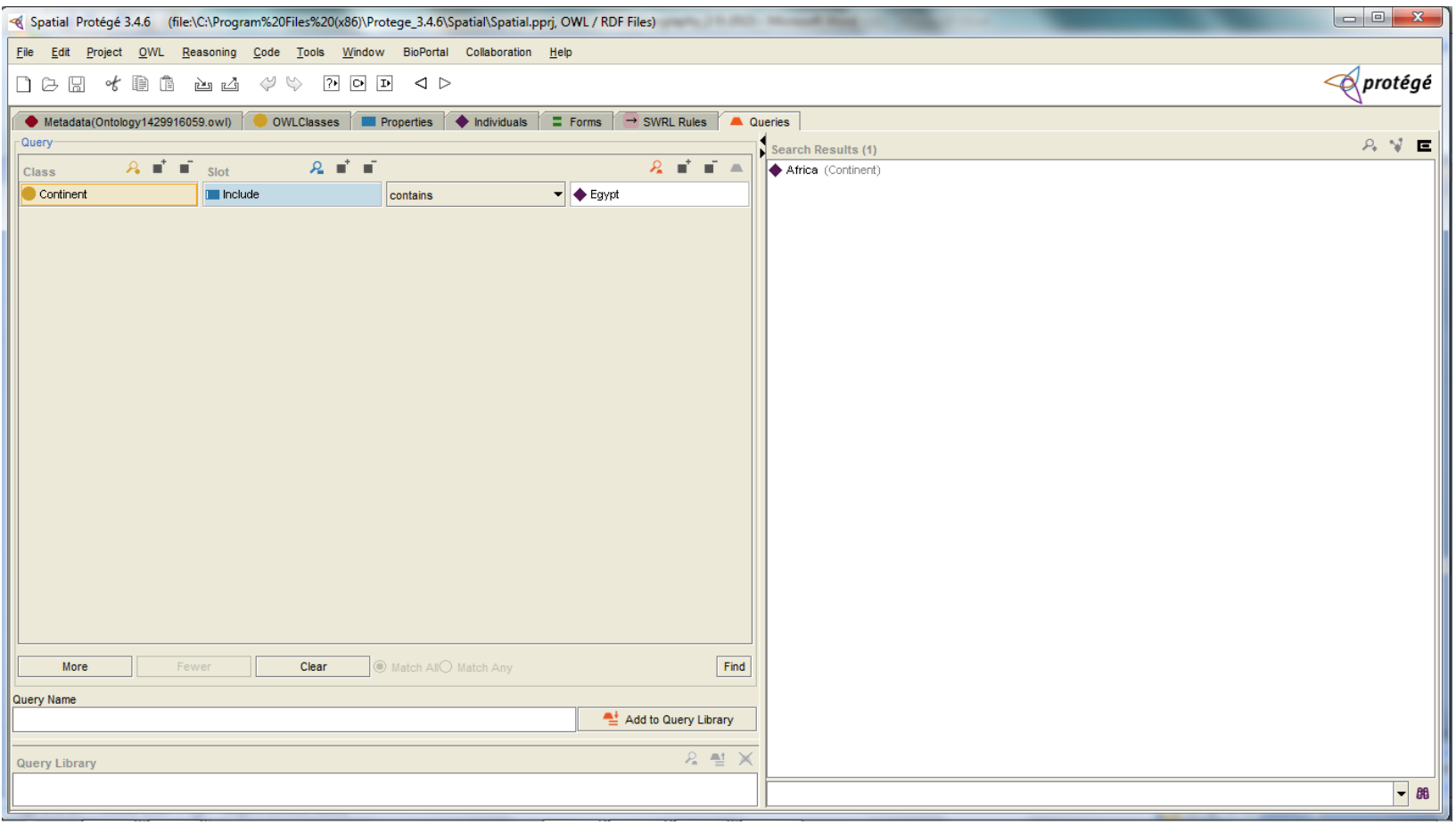

Figure 6: A snapshot of protégé query tool

\section{CONCLUSION AND FUTURE WORK}

In this research, the World geographical ontology model has been developed based on analysis which finds different classes required to represent the ontology. Classes' attributes and relations between different objects have been also specified. The ontology has been formulated using logical form to enable inferring different relations and extracting deeper knowledge. The proposed model has been implemented using protégé tool. The related data has been collected and the model has been tested. Different queries have been tried to test the importance of the proposed ontology to answer users' queries.

In the future, more data can be collected to be represented in the ontology and more knowledge and relations can be inferred from the ontology. And a geographical search engine based on the World ontology could be developed to make use of the ontology to expand the search to reach more meaningful results. It will target geographical researchers to have the ability to execute more intelligent query that can't be implemented in traditional search engine. Where the retrieved geographical information from the ontology can be used to expand the search query through the web to find more relevant resources to the searched location or geographical item.

\section{REFERENCES}

[1] Gaihua Fu, Christopher B. Jones and Alia I. Abdelmoty. "Building a Geographical Ontology for Intelligent Spatial Search on the Web", In Proceedings of IASTED International Conference on Databases and Applications, Springer Verlag, pp: 167-172, 2005.

[2] Cui Wei. "Using Ontology to Achieve the Semantic Integration and Interoperation of the Geography Information System [D]", thesis for doctor's degree, Wuhan university2004.

[3] Yandong Wang, Jingjing Dai, Jizhen Sheng, Kai Zhou, Jianya Gong, "Geo-ontology design and its logic reasoning", Geoinformatics 2007: Geospatial Information
Science, edited by Jingming Chen, Yingxia Pu, Proc. of SPIE Vol. 6753, 675309, (2007).

[4] Song Wei, Zhang Ming. "The compendious tutorial for semantic web", Beijing: higher education publishing company, 2004.

[5] Janowicz, Krzysztof, "Observation-Driven Geo-Ontology Engineering”, Transactions in GIS 16.3 (2012): 351-374.

[6] Xu, Jinghai, Timothy L. Nyerges, and Gaozhong Nie. "Modeling and representation for earthquake emergency response knowledge: perspective for working with geoontology." International Journal of Geographical Information Science 28.1 (2014): 185-205.

[7] Martínez, David Carral, Krzysztof Janowicz, and Pascal Hitzler. "A logical geo-ontology design pattern for quantifying over types." Proceedings of the 20th International Conference on Advances in Geographic Information Systems. ACM, 2012.

[8] Sun, Min, Xiu-wan Chen, and Fei-zhou Zhang. "Geoontology." Geography and Geo-Information Science 3 (2004): 002.

[9] Bharambe, Ujwala, and S. S. Durbha. "Analogy based similarity mining for geo-ontology matching." Geoscience and Remote Sensing Symposium (IGARSS), 2014 IEEE International. IEEE, 2014.

[10] Sinha, G., et al. "Surface Network Ontology Design Patterns for Linked Topographic Data." Manuscript submitted to Semantic Web for review (2014).

[11] Li, Wenwen, Vidit Bhatia, and Kai Cao. "Intelligent polar cyberinfrastructure: enabling semantic search in geospatial metadata catalogue to support polar data discovery." Earth Science Informatics 8.1 (2015): 111-123. 
[12] Kalbasi, Reza, et al. "Collaborative Ontology Development for the Geosciences." Transactions in GIS 18.6 (2014): 834-851.

[13] Campelo, Claudio EC, Brandon Bennett, and Vania Dimitrova. "From polygons and timestamps to dynamic geographic features: grounding a spatio-temporal geoontology." Advances in Conceptual Modeling. Springer Berlin Heidelberg, 2012. 251-260.

[14] Zhu, X.; Chen, D.; Zhou, C.; Li, M.; Xiao, W. "Cardinal Direction Relations Query Modeling Based on GeoOntology." ISPRS-International Archives of the Photogrammetry, Remote Sensing and Spatial Information Sciences 1 (2012): 179-184

[15] Gimenez, Paulo JA, Astério K. Tanaka, and Fernanda Araujo Baião. "A geo-ontology to support the semantic integration of geoinformation from the National Spatial Data Infrastructure." GeoInfo. 2013.

[16] Ying Xia, Shiyan Luo, Xu Zhangand Hae Young Bae "A Novel Retrieval Method for Multimodal Point of Interest Data." International Journal of Multimedia \& Ubiquitous Engineering 9.7 (2014).

[17] Sinha, Gaurav, et al. "An ontology design pattern for surface water features." Geographic Information Science. Springer International Publishing, 2014. 187-203.

[18] G. Stumme, M. Ehrig, S. Handschuh, A. Hotho, A Maedche, B. Motik, D. Oberle, C. Schmitz, S. Staab, L. Stojanovic, N. Stojanovic, R. Studer, Y. Sure, R. Volz, V. Zacharias. "The Karlsruhe View on Ontologies", Technical Report University of Karlsruhe, Institute AIFB, 2003.

[19] http://protege.stanford.edu/index.html 